\title{
10 Things less spoken - HIV research with adolescent boys and young men
}

\author{
Implications for theory, policy \\ and practice
}

\author{
Lesley Gittings, Rebecca Hodes, Christopher J. \\ Colvin and Nompumelelo Zungu
}

\section{Introduction}

There is an increasing acknowledgement that men and boys must be better engaged in HIV prevention and treatment initiatives. Improving men's access to prevention and treatment services would result in a 'triple dividend', benefitting boys and men themselves, their sexual partners and their families (UNAIDS, 2017).

Although girls and women are biologically and socially more vulnerable to acquiring HIV, men access HIV prevention and treatment services less and at a later stage of disease progression and are more likely to die whilst on ART than women (Cornell, McIntyre and Myer, 2011; Johnson et al., 2013).

The adolescent HIV epidemic presents further challenges. AIDS-related illness is the leading cause of death among adolescents in Eastern and Southern Africa (ESA), and these deaths have tripled among adolescents since 2000 while declining in all other age groups (WHO, 2015). Adolescent boys have significantly lower HIV prevalence in late adolescence and early adulthood than adolescent girls and young women (AGYW). However, adolescent boys experience sharp increases in HIV prevalence in the years that follow, suggesting that adolescence may be a critical time where boys form risk behaviours and are exposed to new vulnerabilities.

Despite a growing number of interventions that work with men and boys, there is limited research that explores the processes, politics, limitations and challenges of such work (Gibbs, Vaughan and Aggleton, 2015). This chapter responds to this gap by presenting some methodological and ethical considerations in working with adolescent boys and young men (ABYM).

The first sections of this chapter provide a theoretical and conceptual overview and outline some current debates within masculinities research and HIV social science. The findings comprises three main components, each detailing a particular occurrence from Gittings' doctoral research (supervised by Hodes and Colvin), which focused on the health beliefs and experiences of ABYM (aged 13-24) living with HIV in South Africa's Eastern 
Cape province. It is a sub-study of Mzantsi Wakho, a mixed-methods, longitudinal community-traced study of adolescents living with HIV $(n=1057)$.

The findings section begins by asking, 'Who is best placed to work with adolescent boys and young men?', highlighting the significance of positionality and intersubjectivity. The section then explores the strategies of engagement used by young male researchers working with adolescent boys. It employs the dual interpretive frameworks of 'hegemonic masculinities' and the conceptualisation of both dominant and alternative masculinities bound up in Xhosa masculine identities. It focuses on how considered, contextually grounded performances of masculinity may encourage boys to move beyond dominant masculine scripts and allow for greater honesty, vulnerability and the enactment of alternative masculine norms. The section then considers potential opportunities, as well as pitfalls and challenges, of interventions and research focusing specifically on ABYM.

This chapter highlights some methodological and ethical considerations for working with this diverse group and challenges dominant assumptions about masculinity.

\section{Theory, concepts and definitions}

This chapter acknowledges the social construction of gender as a binary, which separates certain behaviours and attitudes into those deemed 'feminine' or 'masculine'.

Hegemonic masculinity has been described as the dominant form of an idealised masculinity within a society, which imposes meanings about the position and identity of other forms of masculinity and femininity (Connell, 2005). In many settings, the hegemonic forms of masculinity promote values and practices of independence, physical strength, sexual prowess and restraint in displays of emotion or vulnerability. These values and practices are, in turn, seen to act as powerful barriers to avoiding health risks and managing health problems effectively when they do emerge (Courtenay, 2000). This understanding of the potential links between hegemonic forms of masculinity and poor health has been applied to the field of HIV and health research as a way to understand men's poorer engagement in health services, risky sexual practices and poorer HIV-related outcomes (Gibbs, Vaughan and Aggleton, 2015).

The concept of hegemonic masculinity encompasses diverse behaviours and identities, produced contextually and relationally (Wetherell and Edley, 2014). This chapter recognises the malleability and the multiplicity of masculinities while acknowledging the limitations of the concept. We use hegemonic masculinities as an interpretive framework through which to view the beliefs and behaviours of boys and young men, specifically in relation to their participation in the research at the centre of this chapter. We explore how hegemonic masculine norms were practiced within the study context among Xhosa men and adapted through the processes of engaging with this research project. 
Social scientists in Southern Africa have a rich history of emphasising the importance of context in limiting or enabling the effectiveness of HIV interventions (Hodes and Morrell, 2018). In his theoretical work on Xhosa masculinity, Mfecane (2016) argues that 'Western'1 gender theories are limited in their understanding of Xhosa masculinity, in which the concept of indod $a^{2}$ is central. Similarly, in her work on social representations of AIDS and narratives of risk, Zungu (2013) found that the meanings of some HIV prevention messages as constructed within a 'Western' paradigm may be in conflict with meanings within other contexts. Oyěwùmí (1997) posits that dominant gender narratives and interpretations of the social world 'cannot be assumed uncritically for other cultures' in her writing about her work in Nigeria. The message here is that the ways that gender and masculinity are understood locally must be central to the design and evaluation of research and interventions.

This chapter employs notions of both hegemonic masculinities and Xhosa masculine identities encapsulated within the terms indoda and inkwenkwe to examine how boys and young men perform, reconstruct and resist masculine norms in the study context. Despite some suggested theoretical tensions between Mfecane's work on Xhosa masculinities and Connell's hegemonic masculinit(ies), this chapter employs the work of both scholars, with the understanding that the concept of hegemonic masculinities is expansive enough to include these contextual masculine norms. Hegemonic masculinities may be a useful concept to characterise the construction of gender norms and practices, and in particular the stark distinction between manhood and boyhood within the study context.

This chapter also draws on central tenets of feminism and gender studies, emphasising the multiplicity of gendered power dynamics and their interrelationship with health. The concepts of positionality and reflexivity are also used to reflect on the workings of embedded agency in interactions between researchers and participants. Reflexivity has been described as 'the project of examining how the researcher and intersubjective elements impinge on, and even, transform research' (Finlay, 2002). It is a systematic, conscious reflection on research design, methods and findings to provide insight into the subjective aspects of a study and to enable validation of the experiential data provided by study participants. We use the term 'positionality' to describe aspects of identities including race, gender and class, not as essential qualities but as indicators of relational positions (Maher and Tetreault, 1993).

\section{Background: adolescence, health and masculinity}

Given that adolescence is a period marked by multiple physical and psychosocial changes, this time represents an often risky period in young people's lives. It may also be a crucial time in which to intervene to influence the development of health, gendered and sexual behaviours. A growing evidence base suggests that gender transformative work with men and boys 
has the potential to shift harmful gendered norms and practices that might put men and their partners at risk of contracting HIV and having poor AIDS-related outcomes (Dworkin, Fleming and Colvin, 2015). Gender transformative approaches aim to alter discriminatory gender practices, policies, beliefs and ideas, recognising the benefits that gender-equitable environments have for health (Betron et al., 2012).

It has been theorised that men's poorer health outcomes are a result of hegemonic masculine norms, which have a negative effect on health-seeking and protective behaviours. While acknowledging how hegemonic masculine norms vary by context and shift throughout time, commonalities in global hegemonic masculine constructs include courage, independence and selfsufficiency, toughness, emotional and physical strength, sex with multiple partners, fearlessness and control over other men and women (Courtenay, 2000; Mfecane, 2008; Lindegger and Quayle, 2009). In an attempt to perform to these ideals embedded in hegemonic masculinity, it may be difficult for men to seek or accept healthcare, including HIV prevention, testing and treatment services and other forms of support (Colvin, Robins and Leavens, 2010; Mfecane, 2010). Institutional supply-side barriers relating to the availability and delivery of healthcare services are also a significant factor in men's poorer HIV-related health outcomes (Dovel et al., 2015).

There is a growing literature in the ESA region on childhood and adolescent masculinities that considers the pressures exerted on young males to perform to hegemonic masculine norms in contexts underlain by HIV, disempowerment and poverty (Langa, 2010; Govender, 2011; Hensels et al., 2016). This work highlights the vulnerability and uncertainty of ABYM and the importance of better understanding this group, rather than simply framing them in contrast to vulnerable AGYW. Childhood exposure of ABYM to familial, community and structural violence may be internalised and find expression in harmful performances of masculinity, violence perpetration and behaviours that increase HIV acquisition risk (Jewkes et al., 2011; Mathews, Jewkes and Abrahams, 2011).

These texts, alongside critiques of traditional approaches to behaviour change in the context of HIV, suggest that group participatory identity construction work, and initiatives to address social and community-level factors, are better placed than individual-level initiatives that focus on individual behaviour change as a result. Similarly, a focus on attitudes in behaviour change may result in failure to consider important institutional and structural factors that shape behaviours (Pease and Flood, 2008).

\section{Findings and discussion}

\section{Who is best placed to work with adolescent boys and young men? Positionality and intersubjectivity}

This section focuses on positionality and explores the ways in which intersubjective factors and identities shape HIV work with ABYM. Such factors 
include demographic similarity and difference, personality and context. We suggest that it is important to take stock of the many intersecting factors that will shape interactions with participants, and that reflexivity is crucial in HIV prevention research and programming. We also interrogate the assumption that certain aspects of demographic 'sameness', such as geographic proximity, ethnicity or gender concurrence, mean that such interactions will be simpler. The following story explores how aspects of identity, alongside contextual issues, can shape engagements with ABYM.

The Ezobudoda (translated from isiXhosa as 'manhood things') substudy focused on HIV-positive adolescent boys and young men's engagement with traditional and biomedical health services. While establishing Ezobudoda, Gittings asked the research team for help determining the selection criteria for hiring researchers who would work on this project. After three years of data collection, the research team had extensive experience speaking with adolescents and young people about HIV and sexual and reproductive health. Among key requirements for these positions were the need to be 'good with young people', fluency in local languages and the commitment to the research team as a collective. In addition, the research staff suggested - without prompting - certain demographic requirements.

The researchers should be male, they explained, because there are things that participants would not say to female researchers. Part of their argument was based on their perception that certain things are spoken about only by men. They described how certain traditional practices, including ulwaluko, ${ }^{3}$ a month-long initiation into manhood, are highly secretive and that it is a taboo to discuss them with women. Given that there were things about which Gittings, as a woman, should not have knowledge, they suggested that the male research assistants could also act as ethical advisors and mediators, controlling the flow of secretive information from research participants. Beyond collecting data, the researchers would also be crucial in supporting its interpretation due to their proximity to the topic.

They also suggested that it would be easier for male researchers to build relationships with participants and that that these interviews would generate richer and more honest narratives (for an example image of an interview, see the life history interview in Figure 10.1). This belief was based on the assumption that participants are generally more comfortable sharing intimate information with someone of the same gender, due to the perception that they have shared similar experiences.

That speaking about health may be more difficult for men is not surprising given expressions of physical or emotional vulnerability are delimited by hegemonic masculine norms (Connell, 2005). In order to collect richer and more accurate evidence, it would be important for participants to 'open up' and speak freely. Southern African interventions, such as Stepping Stones, Brothers for Life and One Man Can, are premised on the idea that gender-equitable beliefs and practices are better for the health and well-being of men as well as their families and communities. These interventions are centred on in-depth work with men, usually facilitated by other 


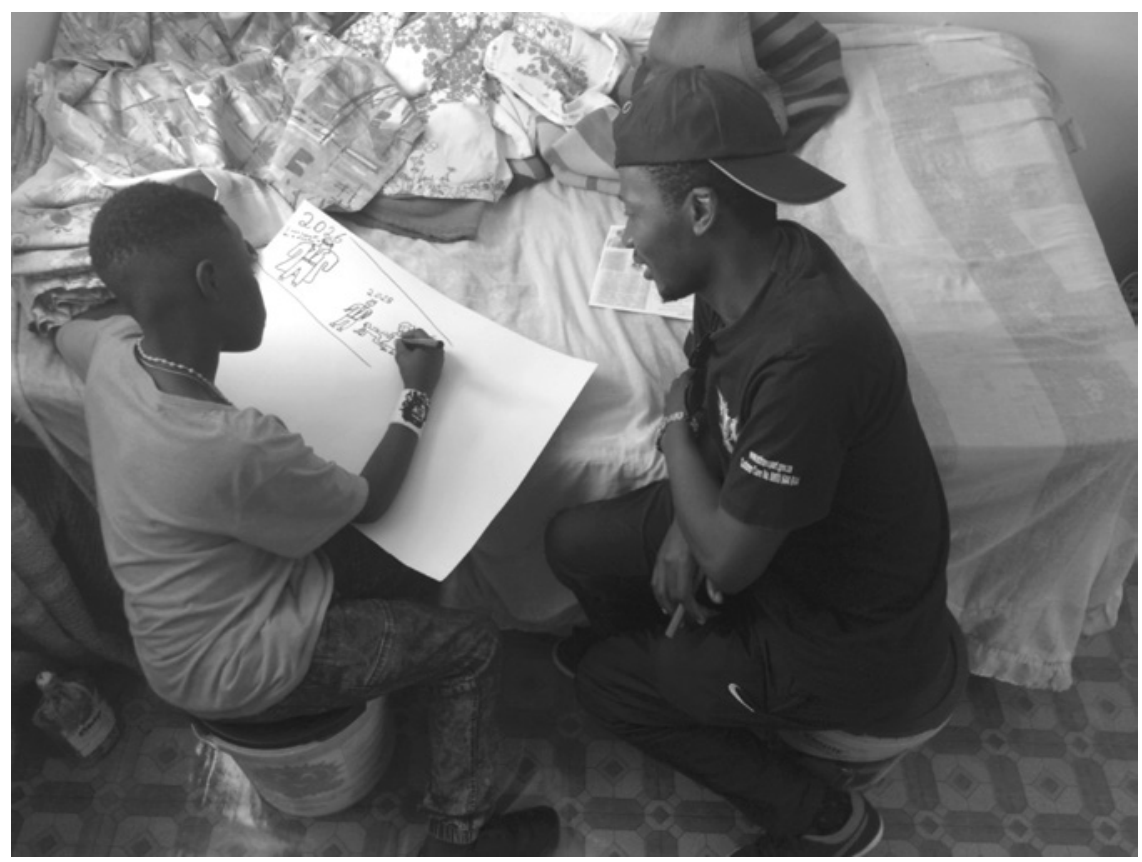

Figure 10.1 Ulwazi (13) conducting a pilot life history interview with researcher Zukolwethu Jantjies, 26 November 2016

men who encourage more gender-equitable practices and enact these practices themselves. They have drawn on certain 'health-promoting' aspects of hegemonic masculinities, such as taking responsibility for the welfare of others (Colvin, Robins and Leavens, 2010). Such campaigns have not been without critique. It has been suggested that certain aspects of these campaigns may reify harmful hegemonic masculine norms (Fleming, Lee and Dworkin, 2014). Despite these potential shortfalls, the lesson we draw here is the way in which positionality may be an important consideration in who does the work with adolescent boys and young men.

A male identity was not the only demographic characteristic advised by researchers as a necessity in their work. They also believed that age was important, and recommended that researchers should be no younger than 25. They reasoned that if the older participants saw the researchers as their peers, they would not be taken seriously. At the same time, they advised that the successful candidates should be no older than 45 , because if participants saw them as elders, they would not feel open to speak frankly to them. They explained that age-based hierarchy is an important factor, especially in a place where issues of sexuality are not usually discussed directly with elders. They also recommended that the candidate(s) should be isiXhosa 
speaking, to circumvent the additional challenges of translation, and that they should themselves be indoda, having followed local traditions and customs of ulwaluko.

What was clear from these discussions was that the research team saw the positionality of the research assistant to be of vital importance and that intersubjective identities were a crucial component of the research and analysis process.

As a result of this planning, the Mzantsi Wakho study hired two 29-year-old isiXhosa-speaking men. Mhlabeni was from Ginsberg, a township directly outside of King William's Town, and Mene was from Dimbaza, a larger urban location a bit further outside of King William's Town. ${ }^{4}$ When the new researchers were deciding which participants they would work with, they requested that they not work with participants in the communities in which they live. They suggested that Mhlabeni interview participants in Dimbaza, where Mene is from, and vice versa. The reason, they explained, was that the social proximity between residents in their communities meant that participants might not feel as safe speaking about intimate issues for fear that others would find out.

In this context, the concern was that participants might be uncomfortable during or after the interview or less likely to open up for fear of unwanted disclosure of their HIV status or personal issues. Unspoken here was also that the researchers may also have been uncomfortable having in-depth and sensitive discussions with fellow community members, as these could complicate personal and professional boundaries. These findings align with evidence suggesting that geographic and social proximity may affect uptake and retention in HIV testing and care services, and that people may choose to visit healthcare providers or institutions in other localities in an attempt to maintain confidentiality (Mhode and Nyamhanga, 2016). This literature contrasts claims that long travel distances to clinics are a key factor in non-adherence. It suggests that in proximate health services, patients fear their HIV status will be disclosed and their confidentiality breached, which may negatively impact on accessing health services.

Researchers calibrated certain types of similarity and difference within the research encounter. Despite believing that linguistic and gendered similarities could support the research aims, they also recommended some distance in terms of age and geographic proximity. In doing so, they challenged a popular assumption that differences between researchers and participants are complicating factors in the research encounter. The next section of this chapter further explores how 'insider' and 'outsider' roles shaped this research.

The ways in which different forms of identity shape interactions was also evident in an unanticipated aspect of the study. As hoped, participants were opening up to the researchers. They were also asking them to act beyond their research role. While drafting the protocol for the study, the ethical and methodological challenges and responsibilities that could be 
encountered were carefully considered, including when participants ask for help. Challenges of working with a group of vulnerable young people in an environment where social services are limited and inconsistent have been documented by Cluver and colleagues (2014). In one week alone, one of the researchers (Mene) encountered a few self-referral cases where ABYM disclosed that they were struggling with drug addiction and requested help. This type of referral was relatively uncommon in the Mzantsi Wakho quantitative survey. Mzantsi Wakho quantitative researchers more frequently encountered participants sharing accounts of violence and hunger, and the established response protocol upheld the ethical commitments and legal requirements of the study. However, accounts of substance abuse were shared more openly and frequently by the participants in the Ezobudoda sub-study. These referrals raised several questions: Was it because the participants were getting older? Was this a gender issue (i.e. more common with boys than with girls)?

Our hypothesis was that while age and gender played a role, it was possible that participants also felt comfortable sharing this type of challenge with Mene because they (rightly) assumed that he would be unlikely to judge or scold them. As someone with a laid-back demeanour and relaxed style, Mene may have given them this impression.

Mhlabeni's referrals were often different in nature. Participants sought assistance for multiple challenges, ranging from intimate subjects such as navigating romantic relationships and material concerns about securing food and work to technology challenges such as how to fix a broken television. Boys frequently asked him for guidance on how to access social services (apply for social grants), and for advice related to circumcision and becoming a man. As someone who appeared to perform to Xhosa notions of being indoda in terms of his presentation, lifestyle and communication style, boys regarded Mhlabeni as an authority on masculine rites of passage.

Cluver and colleagues (2014) suggest that the presence of a caring, attentive adult is a reason why adolescent participants may open up and ask for help. Among study participants, many of whom did not have present fathers, being interviewed by a young man might have also been a rare occasion to have the positive attention of an engaged male figure. The requests for help made by the young men in this study may be attributed to a number of other interrelated factors. The research design saw researchers build relationships with participants over multiple interviews. These encounters also encouraged participants to speak freely and provided opportunities for them to ask questions and direct the conversation based on their interests. An additional factor may have been that participants demonstrated creativity and resilience by leveraging the research encounter as an opportunity to mobilise resources, a phenomenon that has been documented by Vale and Thabeng (2015). In the mix of all of these factors, given the different nature of referral cases that Mhlabeni and Mene met, a number of elements may have played a role in what participants felt comfortable to disclose. These 
may have included participants' perceptions of the researchers' interest in them and how they perceived the researchers' authority, expertise, abilities and personalities.

One finding here, which can be directly applied to HIV prevention research and programming, is that demographic factors coalesce with individual subjectivities and contextual circumstances in shaping interactions between adolescents and adults and between participants and researchers/ programmers. The preceding case study suggests that a close, reflexive consideration of how intersubjective identities operate alongside the research or programmatic aims is warranted in working with adolescent boys and young men. Contextual factors, such as access to social support and engagement with caring adult male figures, may powerfully influence interactions with participants.

Careful consideration should also be given to the contexts and needs of participants and how to respond to requests for support. Researchers and programmers must undertake adequate planning and training prior to beginning a project, so that they are well-equipped to respond to the ethical and methodological imperatives of the work. Managing participant expectations and upholding the ethical commitments made are crucial steps to mitigating any mistrust or disappointment among participants and to guard against producing false expectations and empty promises. This process is especially important when working with adolescent boys and men in contexts of constraint and vulnerability.

\section{Performing masculinit(ies) - strategies for engaging adolescent boys}

These cases in some ways challenge the conventional belief that men do not seek care and support. One can infer that the participants had neither adequate access to social services and resources nor people who could effectively refer them. But was there something beyond the researchers' positionality that allowed participants to express themselves? How did these relationships form? In this section, we explore strategies to create more comfortable spaces for participants and argue that performances of both hegemonic and alternative masculine norms can foster the formation of such key relationships. We document how Mene and Mhlabeni performed to hegemonic masculine norms to gain trust and respect, and how they created spaces for alternative forms of expression by making boys feel comfortable.

While observing the researchers' engagements with participants, it became clear that they curated their performances of masculinity in order to make them feel comfortable and free to share. In the research setting, boys are often subject to instruction by older men and women, and a person is considered to be indoda only after completing ulwaluko. His age as a man begins from this point and is an organising factor that manifests in everyday interactions and traditional ceremonies alike. The process of 
initiation, and the time following, is often marked by instruction and testing by 'older' young men.

At the beginning of our research, 17 participants had undergone ulwaluko, mostly within the last year or two. This meant that even the 'oldest' participants were much younger, and in a different stage of life, than the researchers. The remainder were considered amakhwenkwe (translated from isiXhosa as 'boys') because they had not undergone ulwaluko.

Despite this evident hierarchy, in conversations with participants, the researchers often used descriptors such as 'my man', 'boss', bhuti ('brother' in isiXhosa) and 'my brother', treating participants in a manner that acknowledged their identities as emerging adult men. This approach affirmed participants' masculine identities and conveyed a sense of respect, familiarity, legitimacy and confidence. Although the researchers did not develop friendships per se with participants, they leveraged a different type of social capital that drew on their identities as employed, 'cool' young men to achieve the research aims. Making participants feel comfortable in this way supported the collection of rich data. However, this strategy also posed possible ethical challenges, including blurring boundaries between research and personal relationships and demonstrating simultaneous authority and familiarity that may have made it difficult for participants to set the terms of engagement.

Also relevant here are the ways that researchers perform to 'insider' and 'outsider' roles, in order to encourage participants to speak openly. In her work on Xhosa men's narratives of HIV risk, Zungu (2013) leveraged her outsider role (as woman of Zulu ethnicity) to probe sensitive matters that could not be spoken about in the presence of a Xhosa female. Despite speaking isiXhosa, and having worked for years in her research area, this outsider role allowed her to engage participants as authorities and informers of the subject.

By contrast, Mene and Mhlabeni leveraged their role as insiders by nature of having been legitimised as men by undergoing the process of ulwaluko. However, they also used the authority that having gone through this process bestowed upon them. These masculine performances were employed as a way to legitimise participants' masculine identities and establish familiarity with them. Although seemingly counterintuitive, such performances were the starting point for participants to become comfortable expressing more personal experiences and vulnerabilities. Despite the benefits of this approach, careful consideration should be given to avoiding further entrenching hegemonic masculine gender norms and to minimising social desirability bias.

Many participants in this sample have demonstrated creativity, agency and resilience in moving through precarious and challenging situations. This experience was evident in the ways that boys navigated health facility attendance and medicine-taking, responded to difficult social and familial situations and excelled in multiple areas of their lives. At the same time, 
they faced multiple intersecting challenges and vulnerabilities as (mostly) poor, HIV-positive and 'Black'5 young people, growing up in contexts of precarity and constraint.

That people have lived experiences that are informed by multiple intersecting privileges and marginalisations (Crenshaw, 1991) is relevant here. By engaging with boys' masculine social capital ${ }^{6}$ as an entry point, Mhlabeni and Mene discussed participants' structural, relational and personal vulnerabilities with them.

A methodological lesson from observing the researchers was that through performing to participants' masculinities (an area where participants held power), an entry point was created to express vulnerability. By engaging participants in a way that made them feel comfortable and validated, the researchers created a starting place to encourage them to speak about more challenging aspects of their identities and lives. This approach fits within a multi-level approach to children and resilience - one which considers social, material and relational factors that may affect their well-being while also recognising their agency and life skills (Skovdal and Daniel, 2012).

The ways in which male community health workers engage men living with HIV bear some similarity. In a previous study, we observed that male community health workers acted in a warm and informal way towards their clients and indirectly broached sensitive subjects (Gittings, 2016). In addition to employing these strategies with participants, Mene and Mhlabeni drew on their social capital as 'older' young men who were employed and 'cool'. They performed to hegemonic masculine norms as a way to gain respect and trust from male participants. This careful strategy, when performed properly, often made boys feel comfortable.

This groundwork, in turn, allowed for expressions of vulnerability and emotion - typically performances associated with alternative masculine norms. Participants spoke extensively about personal issues related to health services engagement and intimate relationships and expressed opinions that might be seen as controversial. Heartache, anger and fear were present in many participants' narratives. As discussed earlier, some also asked for advice and help.

While recognising the value of HIV research and programming work with ABYM, we must remain mindful of the pull of patriarchy, which privileges the perspectives and the needs of men above women and gender nonconforming people, and of heterosexual men above gay and transgendered men. Meeting men and boys 'where they are at', while at the same time not reifying gender binaries and harmful gender norms, is a challenge. If the goal is healthier individuals and societies and gender transformation, engaging strategies that draw on masculine norms and identities is not an end in itself but rather a means towards furthering research and programming practice for better health and gender equity outcomes. We explore this in the final section, which focuses on opportunities, pitfalls and challenges of such work. The strategies outlined above engaged aspects of 
hegemonic masculine identity and drew on their authority and resonance in fostering engagements with greater depth, trust and honesty.

Not all participants felt open to, or comfortable with, Mene and Mhlabeni's strategic performances of masculinity. For example, one participant who Mhlabeni believed to be 'gay' (his word) chose not to do a second interview. Another participant whose father was from Ghana openly questioned Xhosa notions of masculinity; Mene responded to this interaction neutrally, but he later disclosed that the experience had bothered him. These situations demonstrate the limitations of gender norms and scripts. In other words, formulaic performances to engage with participants are not always effective, and adolescent boys and young men require engagements that resonate with them.

However, the strategies that Mhlabeni and Mene employed worked relatively well most of the time. Almost all participants were willing to participate in a second and third interview, and some asked - unprompted - for the researchers to come back. In expressing their vulnerabilities, they demonstrated an ability and openness to alternative masculine performances. Their feeling comfortable was in part due to Mene's and Mhlabeni's strategies that drew on the authority offered by hegemonic masculine norms in order to acknowledge them as 'men'. This required delicately deploying hegemonic masculine norms without at the same time further entrenching them. In this context, being indoda by nature of having completed the process of ulwaluko allowed them to occupy the most honoured type of masculinity (Mfecane, 2016).

Outlined above are considerations of positionality, context and strategies for engaging boys and young men in HIV research and programming. The final section traces the contours of some of the debates around work with men and boys by drawing on case studies alongside the substantial and growing academic and programmatic literature that interrogates the meanings, benefits and challenges of such work.

\section{Working with adolescent boys and young men - opportunities, pitfalls and challenges}

While recruiting research assistants, we asked value-based and situational questions with the aim of determining if the interviewees held genderequitable beliefs. Both researchers responded in a way that was in line with the values and skills we were looking for.

Over the period of a year, Gittings, Mene and Mhlabeni took many long drives together to visit participants, during which they would explore different ideas, happenings and theories together and co-analyse emergent findings. In conversations about gender, sex and intimacy they would usually speak indirectly, zig-zagging around the boundaries of the professional and the personal. As they learned more about each other's communication styles, they also began to fill in the spaces of what was not said. After working 
together closely over a period of many months, Mene and Mhlabeni started to demonstrate certain gender-inequitable beliefs.

One day, ten months into working together, Mhlabeni expressed his belief that women aren't good drivers, which surprised Gittings, because he would often ask her to drive. Mene responded by quipping that he didn't see a difference between men's and women's driving abilities. An animated discussion ensued. Gittings asked Mhlabeni why he asked her to drive if, as a woman, he believed she could not be a good driver. 'It's different with you because I'm used to it', he answered.

In a team meeting with a group of approximately 15 researchers, Gittings led an activity as an icebreaker where the team was read a list of yes/no/ maybe questions about beliefs. 'Yes' was signaled by standing up with their arms above their heads, 'no' by sitting down on their chair, and a 'maybe' was displayed by standing with their arms at their sides.

They started with simple statements such as 'I had a nice weekend' and 'It is rude not to greet when you see someone'. After warming up, they engaged with more complex, and potentially more controversial statements. One such statement was 'Women are responsible for cooking and cleaning'. Mhlabeni and Mene stood up and stretched their hands over their heads, while the rest of the team sat down. Everyone else looked up at them, chuckling from their seats - partly in discomfort, and partly because of the irony of the only two staff members directly conducting gender research displaying this belief. The idea that women can be responsible for certain activities is not, in itself, gender inequitable. However, the (unequal) burden of labour and value placed on men's and women's work, combined with the team's different answers when asked about typical 'masculine' responsibilities, meant that these responses likely drew on a genderinequitable belief about women's labour.

Why had they demonstrated this belief, given their awareness about gender equality and their interview responses a year earlier, in which they recounted different perspectives about men's and women's roles? Had their work, though aiming to understand boys' and young men's beliefs and actions, somehow cemented hegemonic masculine norms for them? Did being part of a sub-study that allowed the researchers more flexibility and privileges due to its design reinforce patriarchal norms that men and boys are superior, both as researchers and research participants? Or had the comfort, familiarity and trust the team had built created a space in which they felt open to share their gender-inequitable beliefs? And if the latter was the case, was it a 'good' thing, a step towards, or away from, the gender-transformative objectives of the research?

While not wanting to over-estimate the role of these interactions and their work as researchers on their belief systems, they had actively and continuously engaged about issues of gender over an extended period of time. Their speaking openly about beliefs that were professionally undesirable, and their sharing opinions that were different to Gittings's, suggests that 
relationships had been built where a certain degree of sharing, comfort and ability to express and manage conflicting beliefs was present.

A few days later Gittings asked Mhlabeni and Mene about the opinions they had shared. They said that they felt comfortable displaying their perspectives because, as a function of having frequent and frank conversations about gender, they were more comfortable talking about their beliefs than before. By contrast, they suggested that for the others in the room, their lived practice was also one where women were responsible for domestic work in their homes. They thought that their colleagues may have felt uncomfortable to express their belief that women should be responsible for domestic work in a group setting because they felt it socially unacceptable to do so.

The other potential explanation that Mene and Mhlabeni offered was that their colleagues might hold a different belief in theory, but not in practice, or hold different gendered beliefs for different spheres of their lives - in this case, work and home. This echoes Ratele's (2014) assertion that people might support gender equality in the abstract, but that this might not translate behaviourally. He posits that gender equality discourse may influence men's support of gender equality 'in the abstract' but that 'men's positive, but ambivalent and resistant gender attitudes' prevail (Ratele, 2014, p. 510). In a review of the evidence on work with men and boys, Gibbs and colleagues (2015) point out that such work does not always lead to behaviour changes that support gender equality. The processes and contexts in which work is implemented play a role in intervention effectiveness (Dworkin, Treves-Kagan and Lippman, 2013).

Were Mene and Mhlabeni's choices to display gender-inequitable beliefs in conflict with, ambivalent to or part of a gender-transformative process? Shifting socialised gender norms can be a lengthy and elusive endeavour (Gibbs, Vaughan and Aggleton, 2015). Is performing to socially acceptable gendered beliefs (while not believing them) better than burying genderinequitable beliefs? In terms of Ratele's (2014) assertion that genderequitable beliefs can exist in the abstract but not in practice, was it possible that Mhlabeni and Mene had 'broken the bubble' of the abstract but not shifted into practice? And was this the same as going back to explicit gender-inequitable beliefs or towards conscientization?

These questions and examples are raised as part of broader debates around working with ABYM for HIV prevention. As described earlier, gender inequality is a driver of HIV transmission, and ART initiation and retention in care are mediated by hegemonic masculine norms. For these reasons, questions of gender-equitable belief and practice are important to research and interventions focusing specifically on HIV and ABYM. It has been suggested that it is through practice that new ways of relating are produced (Sideris, 2004), and that it is necessary to create lived experiences of gender equality through structural and policy approaches as well as programmatic initiatives that change attitudes (Levtov et al., 2014). 
As the preceding case studies and reflections demonstrate, the work of gender transformation is neither straightforward nor formulaic. We suggest that approaches that create space for dialogue and reflection, and that focus on processes (rather than solely on endpoints), are important components in the often complicated work of exploring and challenging gender norms.

Finally, as highlighted by scholars such as Mfecane (2016), Zungu (2013), Oyěwùmí (1997) and Hodes and Morrell (2018), the broader gendered context in which HIV work with men and boys takes place is important to consider. How do we engage with context-specific gendered constructs to support men's improved health outcomes? And what does it mean to be working with men and boys for HIV prevention in a context where women and girls are more biologically and socially at risk to HIV acquisition? Scholars such as Gupta et al. (2008) have mapped out the importance of multilayered and systematic approaches in addressing the multiple, overlapping structural and power dynamics that fuel the HIV epidemic. However, work with men and boys may still raise ethical and political considerations, given that it may be seen to compete for resources and attention with work with women and girls. It is therefore critical that gender-transformative research and practice with men and boys be able to articulate how its methods and objectives line up with broader feminist principles and goals.

\section{Conclusion}

Using case studies of HIV research with ABYM, this chapter raises questions about addressing gendered power imbalances and reducing HIV risks and poor health behaviours within this group.

Given the relational nature of gendered power and HIV risk, it is crucial to work with ABYM in order to improve population health outcomes (UNAIDS, 2017). Interventions to reduce the material vulnerabilities of adolescents have demonstrated efficacy in reducing HIV risk behaviours among adolescent girls, but not boys, suggesting the differently gendered relationships between material vulnerability and risky sexual behaviour (Cluver et al., 2013). This is relevant given that HIV risk increases for ABYM as they enter their twenties and thirties. Efforts and strategies for working with ABYM to prevent them from acquiring HIV, and from transmitting it onwards, require further elucidation.

In feminist and participatory research, the processes through which evidence is gathered are important. The lessons derived from this research point to the need for consideration of positionality and intersubjectivity, and the importance of considering the unique and intersecting identities, especially for those living in contexts of racialisation and discrimination, precarity and poverty. We suggest that careful consideration of demographic and personal qualities of facilitators, researchers and programme workers are important. Engagements can be radically shaped by elements 
of 'sameness', 'difference' and geographic proximity. Findings suggest that demographic factors should be carefully considered in deciding who conducts HIV work with ABYM, that sameness is not always more effective and that considerations of positionality are strongly mediated by context. Drawing on the expertise of participants and local researchers and programmers can help navigate these complexities.

Research and programming that aims to conduct in-depth work with ABYM must also consider the intersecting vulnerabilities and privileges of participants. The same structural factors that inform HIV risk will be present in aspects of participants' lives. During project development, consideration of how to respond to participant needs and requests for help that are beyond the context of the programme/research are essential, especially in contexts of precarity and constraint.

This chapter has described strategies for working with ABYM - including partially leveraging hegemonic masculine interactions - to creating the trusting and open relationships that support in-depth research and interventions to promote health and gender-equitable practice. We found that affirming aspects of participant masculine identities can be a starting point for alternative forms of engagement. This process requires delicate consideration of how to make participants feel safe while not reifying harmful hegemonic masculine norms and rigid gender binaries. In the complicated work of exploring and challenging gender norms, creating space for dialogue and reflection and the use of multiple methods to introduce different perspectives may guard against such risks.

Ideally, research and programming should be framed within conducive policy frameworks and a commitment to changing harmful gendered beliefs in practice. The process of meaningfully engaging men for HIV prevention efforts is informed by a variety of factors, including the very gendered power dynamics that are being challenged.

In a context in which little is known about gender transformation, the work of engaging with hegemonic masculine norms is complicated, with 'progress' being difficult to measure. The complexities and dynamics of gender work with ABYM are reflective of broader workplace and societal dynamics. Building and documenting relationships over time that allow for ongoing, continuous and non-judgemental engagement may provide some clues as to the pathways towards gender equity.

This chapter has drawn on case studies with qualitative researchers to consider some ethical and methodological aspects of HIV work with ABYM. In doing so, it aims to contribute to the movement for engaging men and to raise some theoretical, ethical and practical questions to add to our notions of young masculinities and HIV risk. The case studies have aimed to provide a window into how ABYM experience, manage and perform masculinity and to ultimately add depth to what we understand about this group. 


\section{Acknowledgements}

This evidence was gathered within the auspices of a large longitudinal study on medicine-taking in the Eastern Cape, the Mzantsi Wakho study. We are grateful to co-principal investigators Professor Lucie Cluver and Dr Elona Toska, study collaborators at the Universities of Cape Town and Oxford, and the researchers who supported data collection and analysis as well as the research participants for sharing their perspectives and experiences that formed the basis of this research. Thank you also to Mlamli Mayosi for reviewing and providing input into the early drafts of this chapter and to Kaymarlin Govender, Andrew Gibbs and Timothy Quinlan for their detailed review and comments.

Thank you for the financial support provided by Evidence for HIV Prevention in Southern Africa (EHPSA), a DFID programme managed by Mott MacDonald (MM/EHPSA/UCT/05150014). In addition to EHPSA, Lesley Gittings was a supported by the South African National Research Foundation (NRF) Innovation scheme for doctoral student funding, the University of Cape Town AIDS and Society Research Unit (ASRU), the South African Social Science and HIV (SASH) Programme, an initiative funded by the Eunice Kennedy Shriver National Institute of Child Health and Human Development of the National Institutes of Health (Award \#R24HD077976), the South African National Research Foundation travel funding for postgraduate students, and the University of Cape Town's Max and Lille Sonnenberg Scholarship for international travel.

Christopher J. Colvin was supported by the US National Institute of Mental Health and the South African Medical Research Council (SAMRC) [\#1R01 MH106600]. This content is solely the responsibility of the authors and does not necessarily represent the official views of the aforementioned funders.

Data collection support was provided by the Mzantsi Wakho Study, funded by the Nuffield Foundation [CPF/41513], but the views expressed are those of the authors and not necessarily those of the Foundation; Evidence for HIV Prevention in Southern Africa (EHPSA), a UK aid programme managed by Mott MacDonald; Janssen Pharmaceutica N.V., part of the Janssen Pharmaceutical Companies of Johnson \& Johnson; the Regional InterAgency Task Team for Children Affected by AIDS - Eastern and Southern Africa (RIATT-ESA); UNICEF Eastern and Southern Africa Office (UNICEF-ESARO); the International AIDS Society through the CIPHER grant [155-Hod; 2018/625-TOS], the views expressed in written materials or publications do not necessarily reflect the official policies of the International AIDS Society; Oxford University Clarendon-Green Templeton College Scholarship; Claude Leon Foundation [F08 559/C]; the Leverhulme Trust [PLP-2014-095]; the Oak Foundation [R46194/AA001], [OFIL-20057]/GCRF “Accelerating Violence Prevention in Africa”; the University of Oxford's ESRC Impact Acceleration Account [K1311-KEA-004]; and the John Fell Fund [103/757; 161/033]. 


\section{Notes}

1 The limitations of literal and/or simplistic interpretations of geographical terms have been noted by scholars such as the Comaroff and Comaroff (2012), who suggest that power and marginality are important considerations in interpretation.

2 Constructs of masculinity in the research site hold a strong distinction between a 'boy' (in isiXhosa inkwenkwe, 'uncircumcised boy') and a 'man' (in isiXhosa indoda, a traditionally circumcised person), which are defined by whether a person has completed the process of ulwaluko.

3 Referred to throughout this chapter as ulwaluko (isiXhosa), or traditional initiation/circumcision. A rite of passage, ulwaluko is a powerful organising factor in constructions of masculinity among the amaXhosa (Mfecane, 2016; Mager, 1998).

4 Pseudonyms have been used for researcher and participant names throughout this chapter.

5 Racial identifiers, when used within this chapter, are put within quotation marks to denote that these categories are socially constructed. As much as possible, this work aims to avoid reproducing these categories while acknowledging these historical and ongoing constructs when relevant to this study. We aim to consider race-related power alongside other intersecting factors of power and marginalisation such as class identity, foreign status, language, HIV status and age.

6 Putnam (1993, p. 35) defines social capital as 'features of social organizations such as networks, norms and trust that facilitate action and cooperation for mutual benefit'.

\section{References}

Betron, M., Barker, G., Contreras, J. \& Peacock, D., 2012. Men, Masculinities and HIV/AIDS: Strategies for Action. Available at: https://s28691.pcdn.co/wp-content/ uploads/2014/12/Men-Masculinities-and-HIV-AIDS-Strategies-for-Action.pdf.

Cluver, L. et al., 2013. Child-Focused State Cash Transfers and Adolescent Risk of HIV Infection in South Africa: A Propensity-Score-Matched Case-Control Study. The Lancet Global Health, 1, pp. e362-e370.

Cluver, L. et al., 2014. The Cost of Action: Large Scale, Longitudinal Quantitative Research with AIDS-Affected Children in South Africa. In D. Posel \& F. Ross, eds. Ethical Quandaries in Social Research. Cape Town: HSRC Press, pp. 41-56.

Colvin, C.J., Robins, S. \& Leavens, J., 2010. Grounding "Responsibilisation Talk": Masculinities, Citizenship and HIV in Cape Town, South Africa. Journal of Development Studies, 46(7), pp. 1179-1195.

Comaroff, J. \& Comaroff, J., 2012. Theory from the South: Or, How Euro-America Is Evolving Toward Africa. London: Paradigm Publishers.

Connell, R.W., 2005. Masculinities. Cambridge: Polity.

Cornell, M., McIntyre, J. \& Myer, L., 2011. Men and Antiretroviral Therapy in Africa: Our Blind Spot. Tropical Medicine and International Health, 16(7), pp. 828-829.

Courtenay, W.H., 2000. Constructions of Masculinity and Their Influence on Men's Well-Being: A Theory of Gender and Health. Social Science E $M$ Medicine, 50(10), pp. 1385-1401.

Crenshaw, K., 1991. Mapping the Margins: Intersectionality, Identity Politics, and Violence Against Women of Color. Stanford Law Review, 43(6), pp. 1241-1299.

Dovel, K. et al., 2015. Men's Heightened Risk of AIDS-Related Death: The Legacy of Gendered HIV Testing and Treatment Strategies. AIDS, 29(10), pp. 1123-1125. 
Dworkin, S. L., Fleming, P.J. \& Colvin, C.J., 2015. The Promises and Limitations of Gender-Transformative Health Programming with Men: Critical Reflections from the Field. Culture, Health E Sexuality, 17(Suppl 2), pp. 128-143.

Dworkin, S. L., Treves-Kagan, S. \& Lippman, S., 2013. Gender-Transformative Interventions to Reduce HIV Risks and Violence with Heterosexually-Active Men: A Review of the Global Evidence. AIDS Behaviour, 17(9), pp. 2845-2863.

Finlay, L., 2002. Negotiating the Swamp: The Opportunity and Challenge of Reflexivity in Research Practice. Qualitative Research, 2(2), pp. 209-230.

Fleming, P.J., Lee, J. G. L. \& Dworkin, S. L., 2014. "Real Men Don't”: Constructions of Masculinity and Inadvertent Harm in Public Health Interventions. American Journal of Public Health, 104(6), pp. 1029-1035.

Gibbs, A., Vaughan, C. \& Aggleton, P., 2015. Beyond "Working with Men and Boys": (Re)defining, Challenging and Transforming Masculinities in Sexuality and Health Programmes and Policy. Culture, Health $\mathcal{E}$ Sexuality, 17(Suppl 2), pp. 85-95.

Gittings, L., 2016. "When You Visit a Man You Should Prepare Yourself”: Male Community Care Worker Approaches to Working with Men Living with HIV in Cape Town South Africa. Culture, Health E Sexuality, 18(8), pp. 936-950.

Govender, K., 2011. The Cool, the Bad, the Ugly, and the Powerful: Identity Struggles in Schoolboy Peer Culture. Culture, Health E Sexuality, 13(8), pp. 887-901.

Gupta, G. R. et al., 2008. Structural Approaches to HIV Prevention. Lancet, 372(9640), pp. 764-775.

Hensels, I. S. et al., 2016. Do Not Forget the Boys - Gender Differences in Children Living in High HIV-Affected Communities in South Africa and Malawi in a Longitudinal, Community-Based Study. AIDS Care, 28, pp. 100-109.

Hodes, R. \& Morrell, R., 2018. Incursions from the Epicentre: Southern Theory, Social Science, and the Global HIV Research Domain. African Journal of AIDS Research, 17(1).

Jewkes, R. et al., 2011. Gender Inequitable Masculinity and Sexual Entitlement in Rape Perpetration South Africa: Findings of a Cross-Sectional Study B. J. Shea, ed. PLoS One, 6(12), p.e29590.

Johnson, L. F. et al., 2013. Life Expectancies of South African Adults Starting Antiretroviral Treatment: Collaborative Analysis of Cohort Studies. PLoS Medicine, 10 (4).

Langa, M., 2010. Adolescent Boys' Talk About Absent Fathers. Journal of Psychology in Africa, 20(4), pp. 519-526.

Levtov, R. G. et al., 2014. Pathways to Gender- Equitable Men: Findings from the International Men and Gender Equality Survey in Eight Countries. Men and Masculinities, $17(5)$, pp. 467-501.

Lindegger, G. \& Quayle, M., 2009. Masculinity and HIV/AIDS. In P. Rohleder et al., eds. HIV/AIDS in South Africa 25 Years on: Psychosocial Perspectives. New York: Springer-Verlag.

Mager, A., 1998. Youth Identities and Construction of Masculine Identities in Ciskei and Transkei, 1945-1960. Journal of Southern African Studies, 24(4), pp. 653-667.

Maher, F. A. \& Tetreault, M. K., 1993. Frames of Positionality: Constructing Meaningful Dialogues About Gender and Race. Anthropological Quarterly, Feminism and Postmodernism in Anthropology and the Academy, 66(3 Part 2), pp. 118-126.

Mathews, S., Jewkes, R. \& Abrahams, N., 2011. "I Had a Hard Life": Exploring Childhood Adversity in the Shaping of Masculinities Among Men Who Killed an Intimate Partner in South Africa. British Journal of Criminology, 51 (6), pp. 960-977. 
Mfecane, S., 2008. Living with HIV as a Man: Implications for Masculinity. Psychology in Society, 36(Special Issue on Masculinities 2), pp. 45-59.

Mfecane, S., 2010. Exploring Masculinities in the Context of ARV Use: A Study of Men Living with HIV in a South African Village. Johannesburg: University of Witwatersrand.

Mfecane, S., 2016. "Ndiyindoda" [I am a Man]: Theorising Xhosa Masculinity. Anthropology Southern Africa, 39(3), pp. 204-214.

Mhode, M. \& Nyamhanga, T., 2016. Experiences and Impact of Stigma and Discrimination among People on Antiretroviral Therapy in Dar es Salaam: A Qualitative Perspective. AIDS Research and Treatment. https://doi.org/10.1155/2016/7925052

Oyěwùmí, O., 1997. The Invention of Women: Making an African Sense of Western Gender Discourses. Minneapolis: University of Minnesota Press.

Pease, B. \& Flood, M., 2008. Rethinking the Significance of "Attitudes" in Challenging Men's Violence Against Women. Australian Journal of Social Issues, 43, pp. 547-561.

Putnam, R., 1993. The Prosperous Community. The American Prospect, 4(13), pp. 35-42.

Ramose, M. B., 1999. African Philosophy Through Ubuntu. Harare: Mond Books.

Ratele, K., 2014. Gender Equality in the Abstract and Practice. Men and Masculinities, 17(5), pp. 510-514.

Sideris, T., 2004. Men, Identity and Power. A Case Study of the Re-Invention of "Tradition": Implications for Involving Men in Training and Education About Gender. Agenda, 60, pp. 37-41.

Skovdal, M. \& Daniel, M., 2012. Resilience Through Participation and CopingEnabling Social Environments: The Case of HIV-Affected Children in Sub-Saharan Africa. African Journal of Agricultural Research, 11(3), pp. 153-164.

UNAIDS, 2017. Blind Spot: Reaching Out to Men and Boys, Addressing a Blind Spot in the Response to HIV. Geneva: UNAIDS.

Vale, B. \& Thabeng, M., 2015. Mobilising AID(S)? Contesting HIV as a Social and Economic Resource Among Youth in South Africa's Eastern Cape. Journal of Southern African Studies, 41(4), pp. 797-813.

Wetherell, M. \& Edley, N., 2014. A Discursive Psychological Framework for Analyzing Men and Masculinities. Psychology of Men E Masculinity, 15(4), pp. 355-364.

WHO, 2015. Estimates for 2000-2012: Disease Burden. Geneva: WHO.

Zungu, N., 2013. Social Representations of AIDS and Narratives of Risk Among Xhosa Men. Cape Town: University of Cape Town. 